\title{
Out Come of Distal Tibial Malignant Tumor Treated With wide margine Excision Followed by Tibialisation Of Fibula.
}

\author{
Dr. Saikat Sau ${ }^{1}$,Dr Avijit Basak ${ }^{2}$ \\ ${ }^{l}$ Ms Ortho,Dnb Ortho,Mnams,Diploma In Tissue Banking At Nus Singapore Rmo Cum Clinical Tutor, \\ Department Of Orthopaedics, Medical College Kolkata. \\ ${ }^{2}$ Rmo cum clinical tutor at sskm, kolkata, department of orthopaedics
}

\begin{abstract}
Distal tibial tumor are not uncommon, but reconstruction of distal tibial defect after resection of the whole tumor out with sound oncological margine, is very difficult,because of ankle mortis.After resect distal tibial articular surface, its very difficult to reconstruct the ankle mortis.

Distal tibialmegaprosthesis, is very costly.in our study we have done tibialisation of fibula,along with centralization of fibula and arthodesis of distal talofular joint, futher augmentation by distal tibial locking plate applied on bridge mode.

Gap bone defect is a major challenge. Its treatment has evolved over the years from amputation tolimb reconstruction through vascularised graft, distraction osteogenesis and use of customized implants. Availability and affordability of these innovative techniques have always been an additionalchallenge in the developing resource poor countries. We report the use of Tibialization ofIpsilateral fibula first suggested by Hahns in 1884 to bridge a gap of $12 \mathrm{~cm}$ in an 8 year old male, with segmental tibia loss from chronic osteomyelitis. We did an end to end transposition of the ipsilateralfibular into the tibia gap defect in a one stage procedure. This was after eradication of theinfective process of osteomyelitis. He commenced partial weight bearing ambulation in cast at 3months and out of cast ambulation at 18 monthspost surgery. The transposed fibula was $75 \%$ tibialized at 18 monthspost surgery.
\end{abstract}

Conclusion:Fibular is a useful armamentarium in fillingsegmental bone defect.

\section{Introduction}

Primary malignant bone tumours of the distal tibia or the distal fibula are very rare. Amputation has been a standard surgical treatment with satisfactory functional results obtained using an appropriate prosthesis. As the surgery oflimb salvage has developed, reconstruction has been attempted using autograft, allograft, implanted prosthesesand composites.(1-6 )When the ankle is involved, limb salvage presents unique difficulties both in terms of biomechanics and soft-tissue coverage. According to Casadei et al,3arthrodesis is the best reconstructive procedure for the ankle in patients for whom operation for limb salvage isindicated. Ankle replacement arthroplasty has been occasionallycarried out in patients with advanced arthritis.(7-13 )

Medial displacement of fibula with intact all muscular attachment with arthodesis at talofibular junction,and augmentation with distal tibial anatomical locking plate in bridge mode helps in easy skeletal contruct and soft tissue coverage.After 3 month below knee cast immobilization and partial weight bearing from 3 months onwords help in hypertrophy of fibula.as other tarsal joint remain unaffected patient can bear weight easily.

We present six cases with distal tibial malignant tumor treated with excision and biological reconstruction by same side fibula with there follow up.

\section{Material And Method}

Between july 2012and december2016,at West Bengal six patients, three menand three women, with bone tumours of the distal leg hadbiological reconstruction. The mean age at the time ofsurgery was 26 years (11 to 46 ) and the mean follow-up 30months (24 to 60).MRI was carried out on all patients afterplain radiography to determine the extent of each lesion.Each patient had a bone scan, chest radiographs and CT ofthe lungs. The location of the tumours was the distal tibia infive patients and the distal fibula in one.Histopathological diagnosis was obtained by core needle biopsy under local anasthesia;there were four cases of osteosarcoma, and two cases of small blue cell PNET . Allosteosarcomas were stage IIb. Five patients with malignanttumours received neoadjuvant and adjuvant chemotherapyconsisting of ifosfamide, doxorubicin and cisplatin,. The final evaluation included oncologicalinvestigation, the recording of complications andthe evaluation of the functional results, based on the systemof the International Society of Limb Salvage (ISOLS).Regarding vascularisedfibula,it works like a mother bone, any where it can do a miracle.

That's why we planed for medialisation of ipsilateral fibula with out disturbing it's musle attachment. 


\section{Operative techniques}

After proper clinical and all radiological study including metastatic work up we confarm histopathology by core needle biopsy under local anesthesia with only 3 to $5 \mathrm{~mm}$ scar in every case. Which help us during soft tissue coverage in final limb salvage operation, After 3weeks of completion of neoadjuvent chemotherapy we pland for resection of the whole tumor out with proper safe marginethrough as per kwaguchi barrier concept.We use to cut proximal tibia with $3 \mathrm{~cm}$ saftymargine from bone marrow oedema,caused by tumor as per T-1 image of pre neoadjuvent chemo.As articular surface act like a physiological barrier of tumor spraid we dissect out the distal articular surface of tibia.We use to approach through anteromedial longitudinal incision.After proximal tibial cut we send frozen section of proximal tibialmarrow then we progress to distal part for dissection.as soon as frozen section reports give negative margine we cut fibula $2 \mathrm{~cm}$ above the preplanedtibialcut.then distal mortis disarticulate.We dig a square hole on the dome of talus.thenmedialised the fibula reconstruct the diffect after Intramedularryincertion of fibula in the proximal cut margine of tibia.

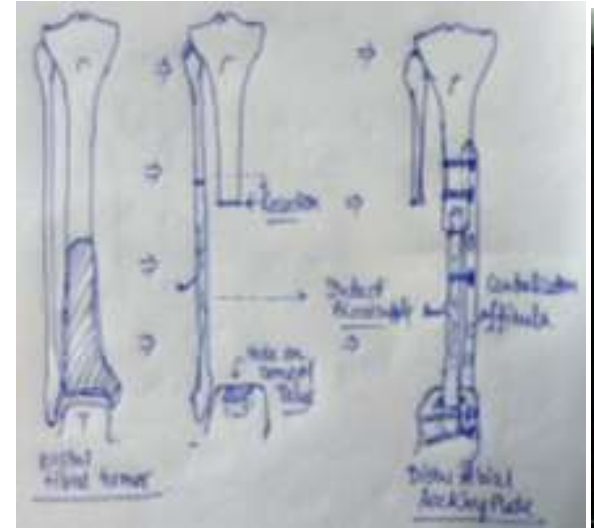

fig-1,

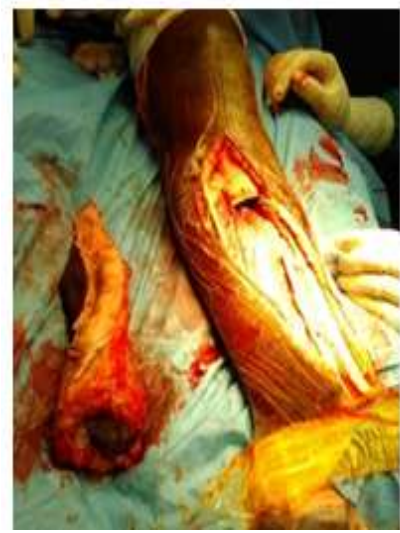

fig-4,

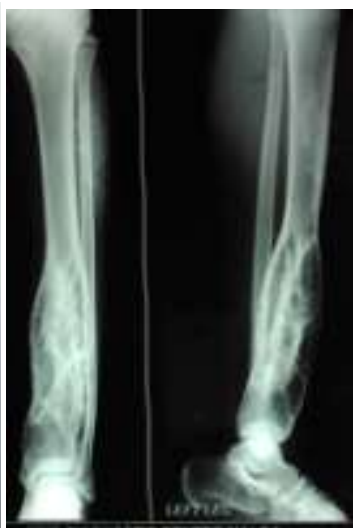

fig-2

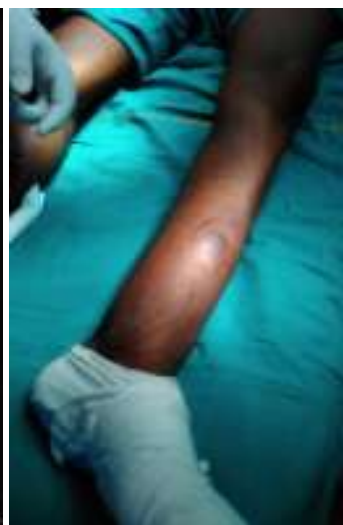

fig-3,
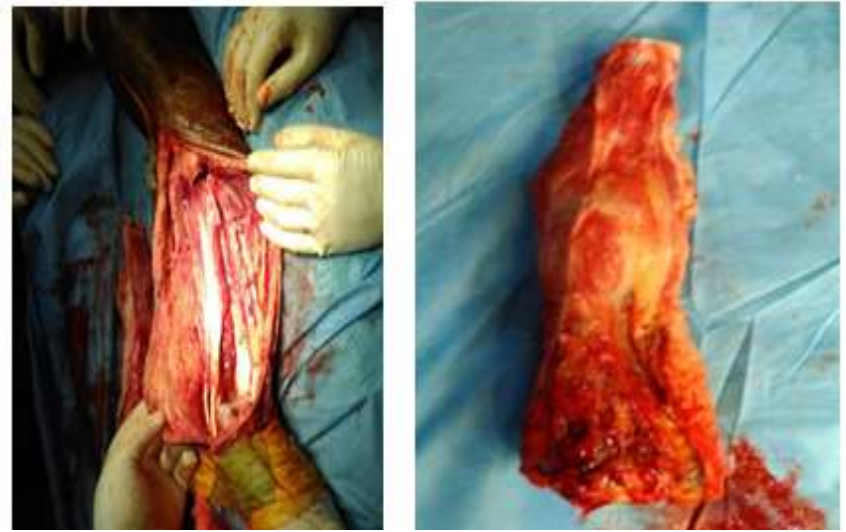

fig-5

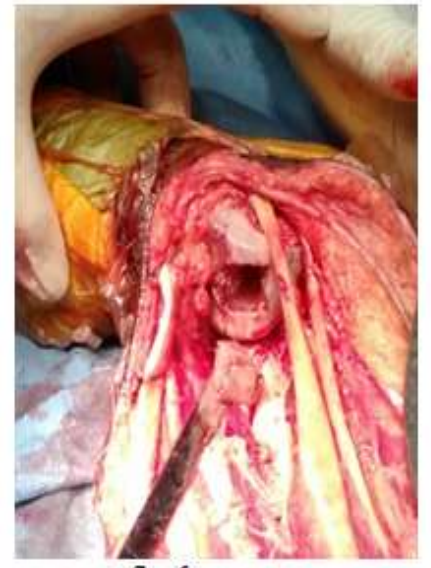

fig-6,

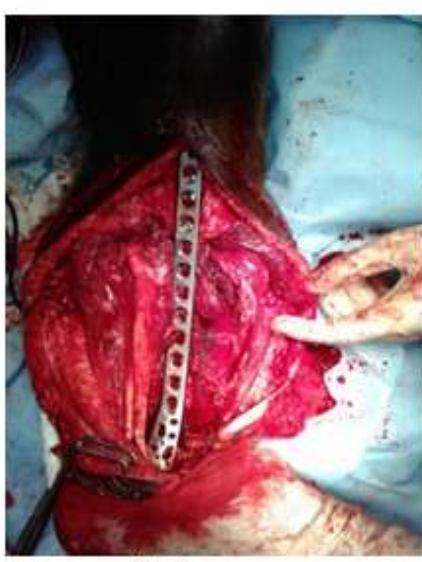

fig-7 

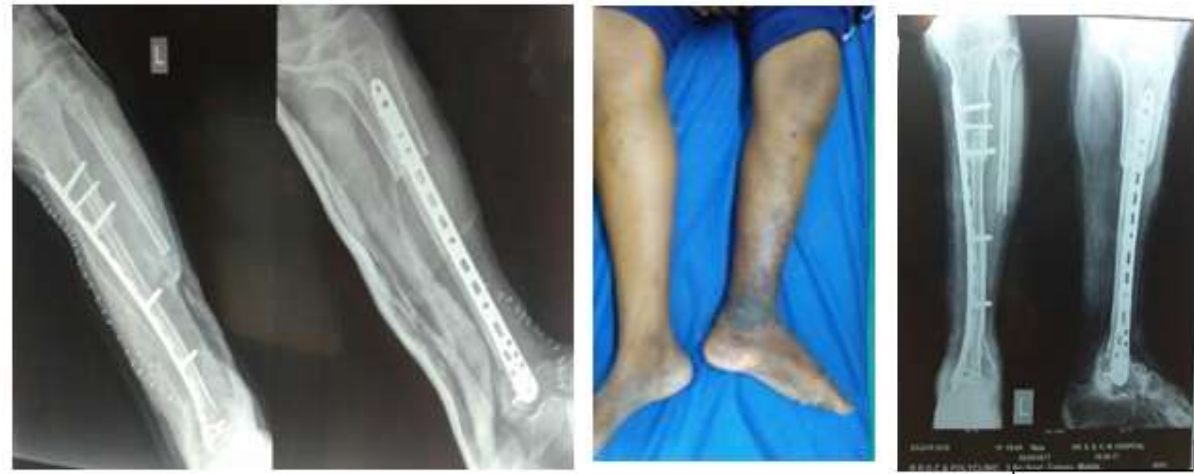

fig-8 fig-9. Fig_10

We further augment the construct with distal tibial anatomical medial locking plate in brigdemode .As there is only one remaining bone in distal part of leg hence soft tissue coverage became quite easy,even after put a locking plate medially.We use to put an negative suction drain every case for atlast 5 days.After 2 weeks we remove stichs and put A short-leg cast for at least three months to resist atendency towards equinus. When the fibula is subjected to more than normalweight bearing stresses, it undergoes hypertrophy andbecomes an integral part of static supporting architectureof the leg. Hypertrophy occurs more commonly when theis mechanically loaded [23,24]. Our rehabilitationschedule did not depend on hypertrophy of the fibula;patients progressed to full weight-bearing once there wasradiological evidence of bony union. Hypertrophy wasseen later after continued weight bearing. The morbidityof this procedure was low regarding frequency and typeof reoperations. We were able to obtain adequate marginswith primary closure of the skin in all our cases anddid not encounter complications like wound dehiscence,deep infections and skin sloughing in any of our patients.

\section{Results}

No local recurrence or distant metastasis occurred in any ofour patients and all remain free from disease at the latestfollow-up. The margin of resection was described as widein three patients and marginal in the other three; histologicalexamination showed the margin to be clear in allspecimen.Pain was minimal; nopatient required analgesics during daily activities.Disturbance of gait was minimal; one patient used a canefor a lengthy walk. The overall functional score (Table I)was $24.2(80.5 \%)$ with a range of 19 (63\%) to 28 (93\%).Compli \begin{tabular}{ll|l} 
of walking (F & Fig-1 -Planning & patients expressed \\
satisfaction to & Fig-2,3-Clinicoradiological &
\end{tabular} Pic Of Distal Tibial PNET

Ca Fig-3,4,5- Resected Specimen And Surgical Defect.

No Fig-6 - Talar Dome

Preparation For Engagement Of Distal Fibula And Talofibulararthodesis. Fig-7 DTALP Augmentation

1. $20 \mathrm{Y}$ Fig-8,9-Post Op Pic

2. $19 \mathrm{Y}$ Fig, 10-fibular hypertrophy ..During wait bearing

3. $25 \mathrm{Y}$

4. $23 \mathrm{Y}$

5. $25 \mathrm{Y}$

$\mathrm{Y}$




\section{$\begin{array}{llllllll}\text { 6. } & 26 \mathrm{Y} & \mathrm{M} & \text { PNET } & \text { INTR COM } & 1 \mathrm{Y} & >90 \% & -\end{array}$}

\section{Discussion}

Below-knee amputation has been the standard surgicalprocedure for malignant bone tumours involving the distaltibia and fibula.(19 )Limb-salvage surgery was rarely consideredfor several reasons. It is difficult to obtain a widemargin of resection because of the limitations of soft-tissuecoverage, but a below-knee prosthesis can provide verysatisfactory function. With the help of advanceschemotherapy havemade such procedures in the distal tibial tumor.Among the many options of reconstruction, arthrodesiswas regarded as best, providing excellent stability of theankle and avoiding problems relating to prosthetic implantation.Casadei et a (13 )reported good functional and oncologicalresults in 12 patients with malignant bone tumoursof the distal tibia, treated by resection and arthrodesis withautogenous bone graft. Complications included infection,limb shortening, fracture.failure. Bishop et al(1) achieved success with a reconstructionusing a vascularised free fibular graft for the treatmentof malignant tumours in the distal tibia.Over a period of five years we have treated nine patientswith malignant tumours of the distal tibia and fibula, usingthis type of reconstruction in five of them. The principalconcerns in the choice of limb-salvage surgery are theextent of soft-tissue invasion and the involvement of thedistal blood vessels. The distal half of the tibia has fewdirect muscle attachments. If neither the posterior tibialartery nor the dorsalis pedis artery proved to be salvageable,amputation was carried out.

Oncologically, all patients remain free from disease, although the resection was marginal in two and theresponse to chemotherapy was less than $80 \%$ in two patients with osteosarcoma. The two disadvantages of this procedure are loss ofmovement at the ankle joint and a little leg length discrepancyin skeletally immature patients. Fortunately,distal epiphysis is not a major contributor to limb length,and the discrepancy is less than that seen around the knee.Moreover, they were well tolerated by our patients, withoutmajor disabilities. We encountered no donor site morbiditylike motor weakness and flexion contracture of the toes, asmuscles originating from the transferred fibula were leftunreleased.The reconstruction of a large defect resulting fromresection of a tumour has always been difficult. Arthrodesiswith centralisation of the fibula is a relatively straightforwardprocedure, requiring no microsurgical expertise, giving durable and satisfactory functional results

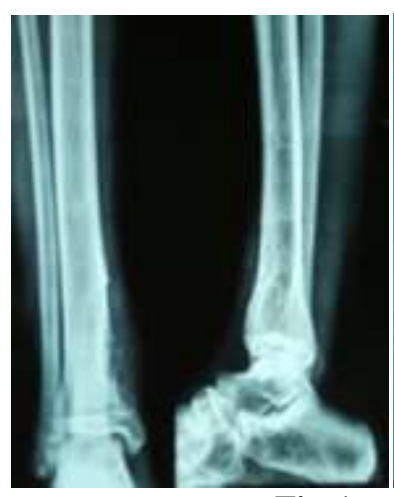

Fig-1,

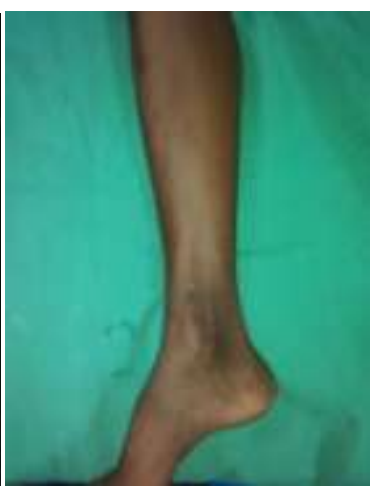

Fig-2

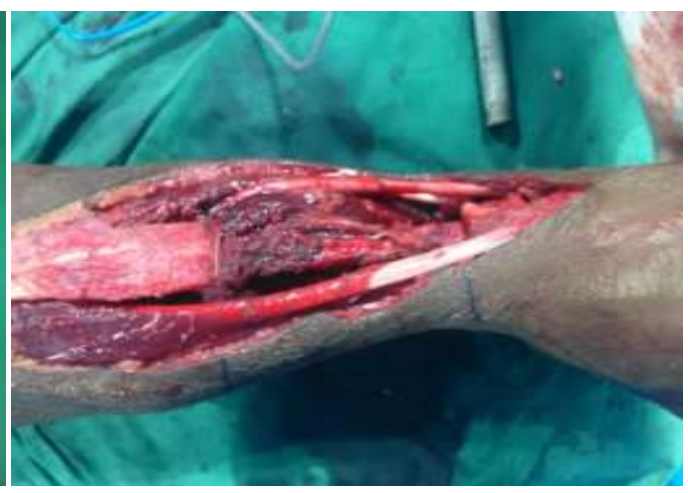

Fig-3, 

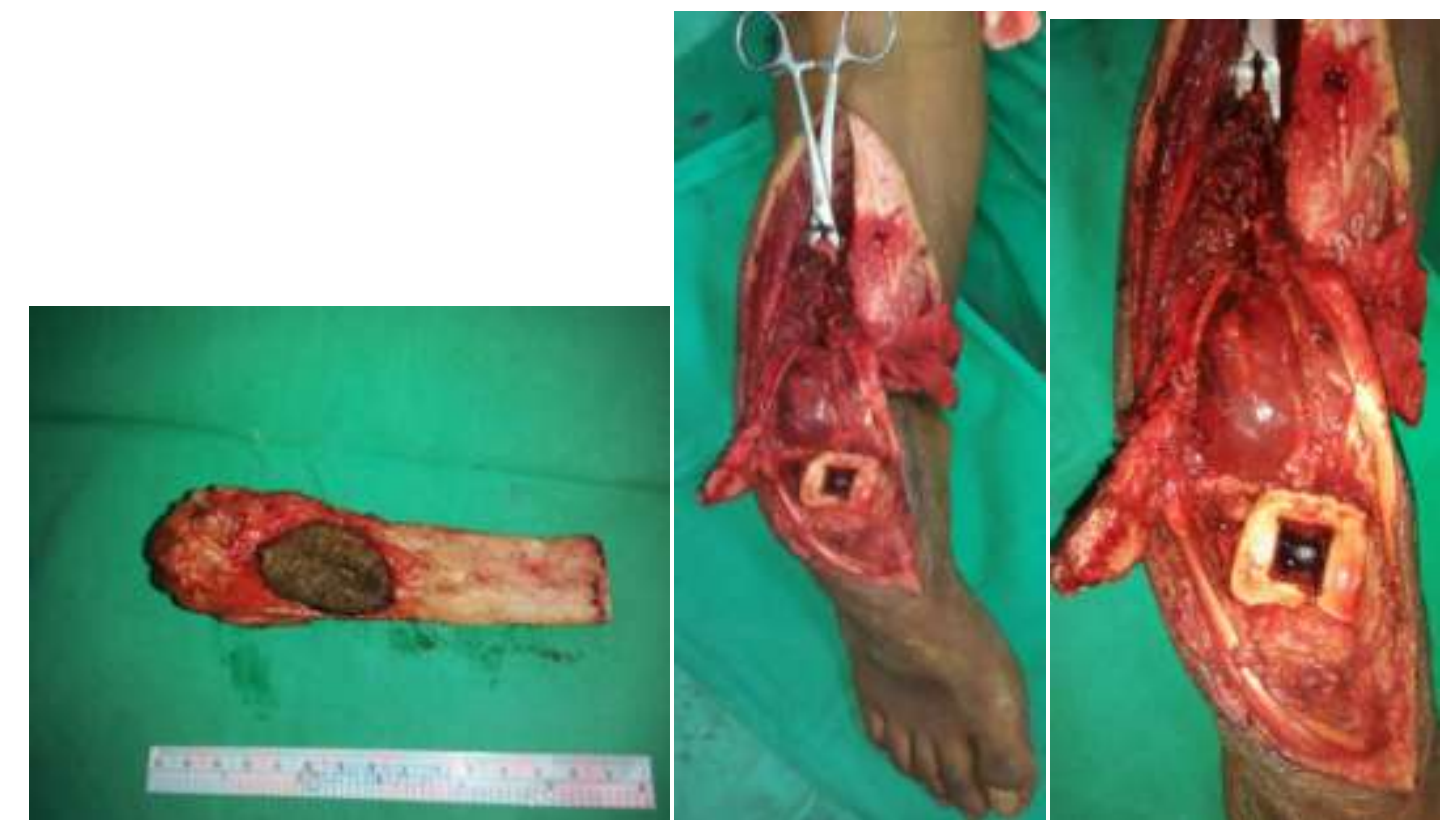

Fig-4,

Fig-5Fig-6

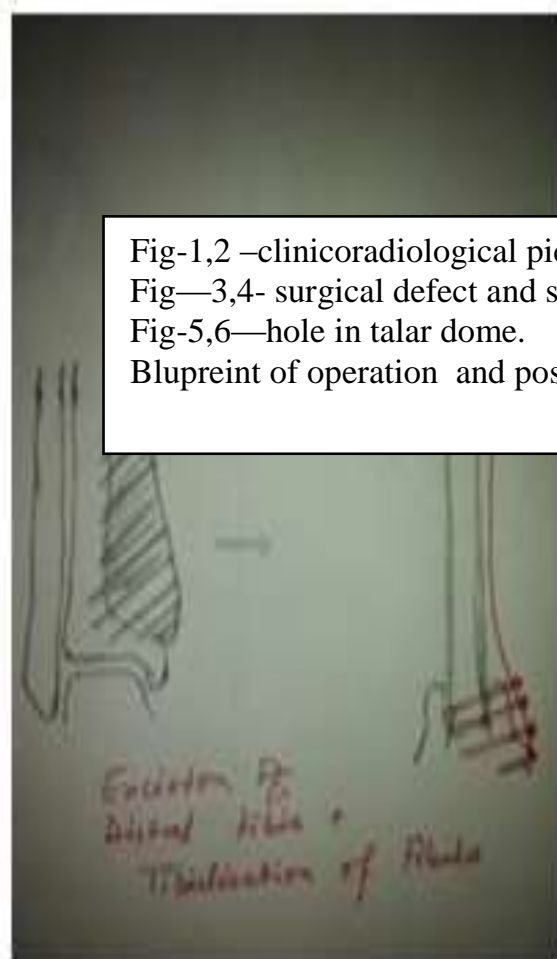

fig-7

Fig-5,6-hole in talar dome.

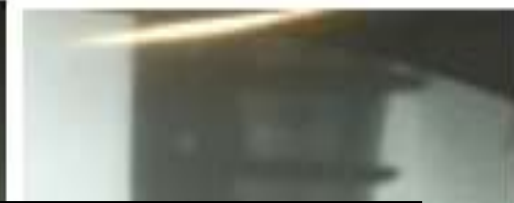

ewings sarcoma

Fig-3,4- surgical defect and specimen

Blupreint of operation and post op costruction.

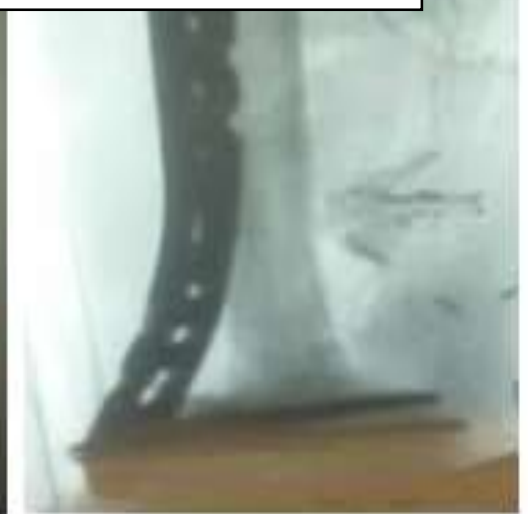

fig,8

\section{Conclusion}

Limb-salvage surgery using a fibula has an acceptable functional outcome with clearance of the tumourin the medium-term analysis of six patients. Larger numbers and a longer follow-up are needed to evaluate theresults of prosthetic reconstruction fairly. Careful patient selection with regard to emotional and 
cosmetic factorsmay justify this type of reconstruction.Distal tibial resection and biological reconstruction is very easy procedure with minimum complications and good long term follow up.

Declearation-No benefits in any form have been received or will be received from a commercial party related directly or indirectly to the subject of thisarticle.

\section{References}

[1]. Bishop AT, Wood MB, Sheetz KK. Arthrodesis of the ankle with a free vascularised autogenous bone graft: reconstruction of segmental

[2]. loss of bone secondary to osteomyelitis, tumor or trauma. J Bone Joint

[3]. Surg [Am] 1995;77-A:1867-75.

[4]. Capanna R, van Horn JR, Biagini R, et al. Reconstruction after resection of the distal fibula for bone tumor. ActaOrthopScand

[5]. 1986;57:290-4.

[6]. Casadei R, Ruggieri P, Giuseppe T, Biagini R, Mercuri M. Ankle resection arthrodesis in patients with bone tumors. Foot Ankle Int

[7]. 1994;15:242-9.

[8]. Choong PF, Sim FH. Limb-sparing surgery for bone tumors: new developments. SeminSurgOncol1997;13:64-9.

[9]. Eckardt JJ, Eilber FR, Rosen G, et al. Endoprostheticreplacementfor stage IIB osteosarcoma. ClinOrthop1991;270:202-13.

[10]. Simon MA, Aschiliman MA, Thomas N, Mankin HJ. Limb-salvage treatment versus amputation for osteosarcoma of the distal end of the

[11]. femur. J Bone Joint Surg [Am] 1986;68-A:1331-7.

[12]. Bolton-Maggs BG, Sudlow RA, Freeman MAR. Total ankle arthroplasty: a long-term review of the London Hospital experience. J Bone

[13]. Joint Surg [Br] 1985;67-B:785-90.

[14]. Hamblen DL. Editorial. Can the ankle joint be replaced? J Bone Joint Surg [Br] 1985;67-B:689-90.

[15]. Kitaoka IIB, Patzer GL, Ilstrup DM, Wallrichs SL. Survivorship

[16]. analysis of the Mayo total ankle arthroplasty. J Bone Joint Surg [Am] 1994;76-A:974-9.

[17]. Kitaoka HB, Patzer GL. Clinical results of the Mayo total ankle arthroplasty. J Bone Joint Surg [Am] 1996;78-A:1658-64.

[18]. Kofoed H, Sørensen TS. Ankle arthroplasty for rheumatoid arthritis

[19]. and osteoarthritis: prospective long-term study of cemented replacements.

[20]. J Bone Joint Surg [Br] 1998;80-B:328-32.

[21]. Lewis G. The ankle joint prosthetic replacement: clinical performance and research challenges. Foot Ankle Int1994;15:471-6.

[22]. Newton SE. Total ankle arthroplasty: clinical study of fifty cases. J

[23]. Bone Joint Surg [Am] 1982;64-A:104-11.

[24]. Unwin P, Cannon S, Kemp H, et al. The Stanmore distal tibial replacement. In: Limb salvage current trends. Proceedings of the 7 th

[25]. ISOLS Meeting, 1993:562

[26]. Abudu A, Tillman RM, Carter SR, Grimer RJ. Endoprosthetic reconstruction of the distal tibia and ankle joint following tumour

[27]. excision. J Bone Joint Surg [Br] 1995;77-B:Suppl 3, 330.

[28]. Bacci G, Picci P, Ruggieri P, et al. Primary chemotherapy and delayed surgery (neoadjuvant chemotherapy) for osteosarcoma of the

[29]. extremities: theIstituto Rizzoli Experience in 127 patients treated preoperatively with intravenous methotrexate (high vs moderate

[30]. doses) and intra-arterial cisplatin. Cancer 1990;65:2539-53.

[31]. Link MP, Goorin AM, Miser AW, et al. The effect of adjuvantchemotherapy on relapse-free survival in patients with osteosarcoma

[32]. of the extremity. New Eng J Med 1986;134:1600-6.

[33]. Mosende C, Gutierrez M, Caparros B, Rosen G. Combination

[34]. chemotherapy with bleomycin, cyclophosphamide and dactinomyci for the treatment of osteogenic sarcoma. Cancer 1977;40:277986.

[35]. Ozaki T, Hillman A, Lindner N, Winkelmann W. Surgical treatment of bone sarcomas of the fibula: analysis of 19 cases. Arch Orthop

[36]. Trauma Surg1997;116:475-9.

[37]. Goorin AM, Andersen JW. Experience with multiagent chemotherapy for osteosarcoma. ClinOrthop 1991;270:22-8.

[38]. Simon MA. Current concepts review: limb salvage for osteosarcoma.

[39]. J Bone Joint Surg [Am] 1988;70-A:307-10.

[40]. Malawer M, Buch R, Reaman G, et al. Impact of two cycles of

[41]. preoperative chemotherapy with intra-arterial cisplatin and intravenous doxorubicin on the choice of surgical procedure for high grade

[42]. bone sarcomas of the extremities. ClinOrthop1991;270:214-22.

[43]. Bos KE, Besselaar PP, Eijken LW, Raaymakers EL (1996) Failure of hypertrophy in revascularised fibula grafts due to stress

[44]. protection. Microsurgery 17:366-370

[45]. Ste' phane S, Eric M, Philippe W, Fe'lix DJ, Raphael S (2009) Resection arthrodesis of the ankle for aggressive tumors of the

[46]. distal tibia in children. J PediatrOrthop 29(7):811-816 